\title{
HARWELL RADIOCARBON MEASUREMENTS VII
}

\author{
A J WALKER, R S KEYZOR*, AND R L OTLET
}

Isotope Measurements Laboratory, Nuclear Applications Centre, Atomic Energy Research Establishment, Harwell, Oxfordshire, OX11 ORA, UK

\section{INTRODUCTION}

The results presented in this list are mostly from our earlier years of operation, the most recent of which were measured in 1984. It is the second of a series of special lists being prepared hopefully to clear the backlog of unpublished dates of this laboratory. All samples are of archaeological origin from the United Kingdom; most have originated from "rescue" type excavations and all were sponsored by the Ancient Monuments Laboratory of the Historic Buildings and Monuments Commission.

All samples were measured by liquid scintillation counting (Otlet \& Warchal, 1978), and the error term quoted is the 1 sigma standard deviation estimate of the full replicate sample reproducibility (Otlet, 1979). The list was produced, as was the last one, from computer-prepared data using procedures described in Otlet and Walker (1983). Calculations are based on the Libby half-life of 5568 years, using NBS oxalic acid standard $(\times 0.95)$ as "modern," both values treated as constants, with $\mathrm{AD} 1950$ as the reference year. All results are corrected for fractionation according to the quoted $\delta^{13} \mathrm{C}$ (wrt PDB) values measured in this laboratory.

\section{ACKNOWLEDGMENTS}

We wish to acknowledge the work of our colleagues G A Bradburn and D G Humphreys with the laboratory measurements and of E F Westall, S E Hasler, and M Gibson with the preparation of the data.

\section{ARCHAEOLOGIC SAMPLES}

\section{England}

\section{St Aldates series}

Wood and charcoal, from St Aldates, Oxford (51 $44^{\prime} 46^{\prime \prime} \mathrm{N}, 1^{\circ} 15^{\prime} 20^{\prime \prime}$ W, Natl Grid Ref SP 514058). Coll and subm by B G Durham, Oxford Archaeol Unit. See Durham $(1977,1983,1984)$ for report on site at $65 \mathrm{St}$ Aldates; for other dates from area, see Harwell I (R, 1974, v 16, no. 2, p 179-180).

HAR-717. SPL319

$870 \pm 70$

$\delta^{13} C=-25.7 \%$ 1977).

Charcoal, id. as charred twigs, from 79-80 St Aldates (Durham, 
HAR-718. SPL406

$$
\begin{array}{r}
1150 \pm 90 \\
\delta^{13} C=-25.4 \% 0
\end{array}
$$

Wood from 79-80 St Aldates (Durham, 1977).

HAR-5339. OX65A313

$$
\delta^{13} C=-28.5 \%
$$

Wood, AML 826484, from early silting after abandonment of ford, trench III (1981), at 65 St Aldates. Coll Dec 1981 and subm Dec 1982. Comment (BGD): result of AD 1100 expected for this material which is from first $0.3 \mathrm{~m}$ silting above abandoned 'Oxen Ford'; this is confirmed by ${ }^{14} \mathrm{C}$ result.

HAR-5340. OX65A317

$1020 \pm 70$

Wood, AML 826485, from core of stone ford, trench III (1981), at 65 St Aldates. Coll Dec 1981 and subm Dec 1982. Comment (BGD): 'Oxen Ford' is thought to have existed from ca AD 800; this result presumably relates to repair or general widening of upstream edge.

HAR-5341. OX65A402

$$
\begin{array}{r}
1080 \pm 70 \\
\delta^{13} C=-29.3 \% 0
\end{array}
$$

Wood, AML 826486, from waterfront at rear of tenement fronting 65 St Aldates, (trench IV, 1982). Coll June 1982 and subm Dec 1982. Comment (BGD): topographically, this feature was assumed to be 13th century AD, however, result is one of earliest dates in Oxford and at that time area was assumed to be broad, forded river channel. Sample came from salvage operation so no other evidence is available.

HAR-5343. OXTMS31

$$
1080 \pm 80
$$$$
\delta^{13} C=-27.4 \%
$$

Wood, AML 826488, from stake at base of gully in S trench at 89-91 St Aldates. Coll Dec 1981 and subm Dec 1982.

\section{HAR-5346. OXTMS35}

$$
\begin{array}{r}
\mathbf{9 8 0} \pm \mathbf{7 0} \\
\delta^{13} C=-27.9 \% \text {. }
\end{array}
$$

Wood, AML 826491, from wooden fender in front of gravel waterfront at 89-91 St Aldates. Coll July 1982 and subm Oct 1982.

\section{Wetwang series}

Samples from sites VI, VII, IX and XII at Wetwang Slack, East Yorkshire. All samples coll by J S Dent or J D Dawes and subm by J S Dent, Humberside Co Council, Beverley, North Humberside. For descriptions of different aspects of site, see Dent $(1978,1978,1982)$; for summary of excavations, 1964-1980, see Dent (1983).

HAR-1665. WE1 AF

$$
\begin{array}{r}
\mathbf{2 1 1 0} \pm \mathbf{8 0} \\
\delta^{13} C=-22.0^{\%} \%
\end{array}
$$

Human bone, AML 760801, from woman's skeleton, burial 155, in Iron Age grave at site VII $\left(54^{\circ} 1^{\prime} 41^{\prime \prime} \mathrm{N}, 0^{\circ} 33^{\prime} 20^{\prime \prime} \mathrm{W}\right.$, Natl Grid Ref SE 
946601). Coll April 1976 and subm May 1976. Comment (JSD): two bronze bracelets, bronze brooch, bronze earring, and glass bead necklace were found with sample.

\section{HAR-1878. WSVI F1AK}

$$
\begin{array}{r}
\mathbf{3 4 5 0} \pm \mathbf{9 0} \\
\delta^{13} C=-25.0 \%
\end{array}
$$

Wood, AML 760784, ca 50\% id. by C A Keepax as oak and hawthorn type, probably mature wood, from remains of coffin at site VI (54 $4^{\circ} 1^{\prime} 41^{\prime \prime} \mathrm{N}$, $0^{\circ} 33^{\prime} 15^{\prime \prime} \mathrm{W}$, Natl Grid Ref SE 947601). Coll April 1975 and subm Sept 1976. Comment (JSD): coffin was from grave thought to be Bronze Age although among Iron Age square barrows; latter is confirmed by ${ }^{14} \mathrm{C}$ result . Inhumation was assoc with animal shoulder blade, possibly deer, and grave fill clearly cut cremation (assumed to be Bronze Age also).

\section{HAR-1879. WSVI F455NS}

$3160 \pm 90$

Wood, AML 760783, from post pipe of circle of nine large posts $\left(54^{\circ}\right.$ 1' 44" N, $0^{\circ} 33^{\prime} 20^{\prime \prime} \mathrm{W}$, Natl Grid Ref SE 946602). Coll Sept 1975 and subm Sept 1976. Comment (JSD): post circle, ca $9 \mathrm{~m}$ diam, more probably from ritual or funerary monument than domestic structure.

HAR-2771. WT 125CR

$2140 \pm 80$

$$
\delta^{13} C=-21.6^{\prime \prime} \%
$$

Human bone, AML 781 193, burial 236, from grave in Iron Age cemetery at site IX $\left(54^{\circ} 1^{\prime} 38^{\prime \prime} \mathrm{N}, 0^{\circ} 33^{\prime} 31^{\prime \prime} \mathrm{W}\right.$, Natl Grid Ref SE 94460()). (ioll June 1977 and subm May 1978. Comment (JSD): bronze bracelet, iron brooch, and glass bead necklace found with burial.

\section{HAR-2776. WSVI F334MD}

$1790 \pm 70$

Human bone AML 781194, burial 117 , from grave in Iron A tery at site VI $\left(54^{\circ} 1^{\prime} 44^{\prime \prime} \mathrm{N}, 0^{\circ} 33^{\prime} 20^{\prime \prime} \mathrm{W}\right.$, Natl Grid Ref SE 9466() 2$)$. (ioll Oct 1975 and subm May 1978. Comment (JSD): iron brooch and pig bones were found with burial.

\section{HAR-2777. WSV1 F292JU}

$$
\begin{array}{r}
\mathbf{2 0 8 0} \pm \mathbf{8 0} \\
\delta^{13} C=-2 l . \sigma^{\prime \prime} /(11)
\end{array}
$$

Human bone, AML 781196, burial 98, from Iron Age grave at Site VI (54 $4^{\prime} 44^{\prime \prime} \mathrm{N}, 0^{\circ} 33^{\prime} 20^{\prime \prime} \mathrm{W}$, Natl Grid Ref SE 946602). Coll Aug 1975 and subm May 1978. Comment (JSD): burial contained sword and shicld.

\section{HAR-4425. WG218EC}

$$
\begin{array}{r}
2270 \pm 100 \\
\delta^{13} C=-26 .()^{\prime \prime}(w)
\end{array}
$$

Charcoal, id. as oak (Quercus sp) and blackthorn (Prunus sp) from mature timbers, also hawthorn (Rosaceae subfamily Pomoideae) and havel/ alder (Corylus/Alnus sp) from twigs or branches, found in post hole in carly Iron Age round house at Site XII (54 $4^{\circ} 1^{\prime} 41^{\prime \prime} \mathrm{N}, 0^{\circ} 33^{\prime} 38^{\prime \prime} \mathrm{W}$, Natl (irid Ref SE 94286010). Coll May 1980 and subm Feb 1981. Comment (JSI)): sample from one of many round houses representing, with post squares, buildings 
of early Iron Age linear settlement. Other assoc features include linear ditches, trackways, and large cemetery.

\section{HAR-4427. WG7AX}

$$
\begin{array}{r}
\mathbf{3 7 8 0} \pm 70 \\
\delta^{13} \mathrm{C}=-25.4 \% 0
\end{array}
$$

Wood, id. by C A Keepax as oak (Quercus sp) from mature timbers, from timber lining of burial cist, burial 12, in early Bronze Age grave at Site XII $\left(54^{\circ} 1^{\prime} 38^{\prime \prime} \mathrm{N}, 0^{\circ} 33^{\prime} 37^{\prime \prime} \mathrm{W}\right.$, Natl Grid Ref SE 943600). Coll May 1980 and subm Feb 1981. Commenl (JSD): grave contained crouched burial with pig bones and very fine battle axe. Only Yorkshire battle axe burial found since advent of ${ }^{14} \mathrm{C}$ dating.

\section{HAR-4428. WE47IUW}

$$
\begin{array}{r}
4260 \pm 80 \\
\delta^{13} C=-25.6 \%
\end{array}
$$

Wood, id. by C A Keepax as oak, (Quercus sp) from mature timbers, from pit at center of interrupted circular ditch at site VII $\left(54^{\circ} 1^{\prime} 41^{\prime \prime} \mathrm{N}, 0^{\circ}\right.$ 33' 26" W, Natl Grid Ref SE 945601). Coll Feb 1977 and subm Feb 1981. Comment (JSD): no bone was recovered from central pit, but monument is probably ritual or funerary; for comparable examples, see Allen (1981).

General Comment (JSD): Wetwang Slack is dry valley in Yorkshire Wolds containing many prehistoric burial and settlement sites. ${ }^{14} \mathrm{C}$ dating has proved useful in identifying second millennium BC features. However, it has not been possible to provide absolute dates for various stages of development of Iron Age cemetery as time-span of its use is short in comparison with precision of ${ }^{14} \mathrm{C}$ dates (see HAR-1665, -2771, -2776, -2777). This is where precision $> \pm 1 \%$ is needed for effective archaeological interpretation.

\section{Hereford series}

Wood from Saxon sites in City of Hereford, Hereford and Worcester. Coll by R Shoesmith, City of Hereford Archaeol Comm. For other results in series, see Harwell III (R, 1979, v 21, no. 3, p 373).

HAR-1735. HE741

$$
\begin{array}{r}
750 \pm 70 \\
\delta^{33} \mathrm{C}=-27.6 \% 0
\end{array}
$$

Wood, AML 753184, from part of timber structure of unknown nature in disused Saxon defensive ditch at City Arms site $\left(52^{\circ} 3^{\prime} 22^{\prime \prime} \mathrm{N}, 2^{\circ}\right.$ 42' 58" W, Natl Grid Ref SO 50913999). Coll 1974 and subm May 1976 by R Shoesmith. Commenl (RS): result shows ditch stayed open and was re-used (Shoesmith, 1982).

HAR-5066. SAMPLF

$$
\delta^{\prime 3} \mathrm{C}=\begin{array}{r}
980 \pm 70 \\
-24.9 \%
\end{array}
$$

Wood, AML 819956, from timber 'corderoy' road $2 \mathrm{~m}$ below present level in King St $\left(52^{\circ} 3^{\prime} 14^{\prime \prime} \mathrm{N}, 2^{\circ} 43^{\prime} 1^{\prime \prime} \mathrm{W}\right.$, Natl Grid Ref SO 50853981). Coll Oct 1980 and subm June 1982 by $D$ Haddon-Reece, Ancient Monuments Lab, London. Comment (DHR): sample came from point where road 
crossed marshy area some $50 \mathrm{~m}$ wide to $W$ of Cathedral. King St appears to be original E-W rd of Saxon town and may have provided $W$ limit of original cathedral nucleus (Shoesmith, 1980), ${ }^{4} \mathrm{C}$ result is of value in considering origin of street plan of Hereford.

\section{HAR-4399. EXG027}

$$
\delta^{13} C=\begin{array}{r}
3520 \pm 80 \\
-22.1 \% 0
\end{array}
$$

Human bone, from possible Bronze Age inhumations at Exning, Suffolk $\left(52^{\circ} 14^{\prime} 45^{\prime \prime} \mathrm{N}, 0^{\circ} 23^{\prime} 18^{\prime \prime} \mathrm{E}\right.$, Natl Grid Ref TL 63036357). Coll by E Martin and subm Feb 1981 by $P$ Murphy. Commenl (PM): result confirms Bronze Age date for burials.

\section{HAR-4473. SKT1155}

$$
\begin{array}{r}
\mathbf{2 4 7 0} \pm \mathbf{7 0} \\
\delta^{13} C=-25.7 \% 0
\end{array}
$$

Charcoal, from contents of possibly Neolithic pot in small pit under platform of Medieval moated site, SKT 011, Stowmarket, Sulfolk $\left(52^{\circ} 10^{\prime}\right.$ $40^{\prime \prime} \mathrm{N}, 1^{\circ} 0^{\prime} 48^{\prime \prime} \mathrm{E}$, Natl Grid Ref TM 060576). Coll by E Martin and subm Feb 1981 by P Murphy, Centre for East Anglian Studies. Comment (PM): pot thought to be from Ardleigh series of Bronze Age urns although rim is unusual. For summary, see Martin (1981).

\section{Mar Dyke series}

Samples from Ardale excavations along line of A13 trunk road, Stifford, Essex, coll by T J Wilkinson, Essex Co Council. See Wilkinson (1980) for description of site.

\section{HAR-4522. 575C-I}

$$
\begin{array}{r}
\mathbf{5 7 4 0} \pm \mathbf{8 0} \\
\delta^{13} C=-27.6 \%
\end{array}
$$

Wood, AML 811636, from bridge pier foundations in valley of Mar Dyke (51 $29^{\prime} 47^{\prime \prime} \mathrm{N}, 0^{\circ} 16^{\prime} 35^{\prime \prime} \mathrm{E}$, Natl Grid Ref TQ 580800). Coll and subm June 1981 by $T \mathrm{~J}$ Wilkinson. Comment $(\mathrm{TJ}$ W): sample from deep sec through Flandrian transgression sequence of peats, plus some humic muds, fine silt clay near base of sequence at $4.60 \mathrm{~m} O D$.

HAR-4523. 575C-II

$$
\begin{array}{r}
\mathbf{4 6 5 0} \pm \mathbf{9 0} \\
\delta^{13} C=-27.9 \%
\end{array}
$$

Wood, AML 811638, from peat containing abundant wood fragments from base of same deposit as $575 \mathrm{C}-\mathrm{III}$ (HAR-4524), $3.60 \mathrm{~m} \mathrm{OD}\left(51^{\circ} 29^{\prime} 47^{\prime \prime}\right.$ N, $0^{\circ} 16^{\prime} 35^{\prime \prime} \mathrm{E}$, Natl Grid Ref TQ 580800 ). Coll and subm June 1981 by T J Wilkinson.

\section{HAR-4524. 575C-III}

Wood, AML 811640, from top of same deposit as 575C-II (HAR4523), 2.65m OD (51 $29^{\prime} 47^{\prime \prime} \mathrm{N}, 0^{\circ} 16^{\prime} 35^{\prime \prime} \mathrm{E}$, Natl Grid Ref TQ 580800). Coll and subm June 1981 by $\mathrm{T} J$ Wilkinson. 
HAR-4525. 575C-IV

$1540 \pm 80$

$\delta^{13} \mathrm{C}=-28.4 \%$

Wood and peat, AML 811641 , from humidified peat and very humic silt mud with occasional wood fragments at $1.20 \mathrm{~m} \mathrm{OD}\left(51^{\circ} 29^{\prime} 47^{\prime \prime} \mathrm{N}, 0^{\circ} 16^{\prime}\right.$ $35^{\prime \prime}$ E, Natl Grid Ref TQ 580800). Coll and subm June 1981 by T J Wilkinson. Comment (TJW): same level as scatter of charcoal fragments in peats (possible clearance or human influence).

HAR-4526. 575C-V

$$
\begin{array}{r}
1470 \pm 80 \\
\delta^{\prime 3} C=-28.5 \%
\end{array}
$$

Peat, AML 811639 , from humified peat of well-developed sub-angular blocky structure $\left(51^{\circ} 29^{\prime} 47^{\prime \prime} \mathrm{N}, 0^{\circ} 16^{\prime} 35^{\prime \prime} \mathrm{E}\right.$, Natl Grid Ref TQ 580800). Subm June 1981 by. T J Wilkinson. Comment (TJW): uppermost sample taken from Mar Dyke sequence ca $70 \mathrm{~cm}$ below modern floodplain surface. Sample is consistent with sequence, see 575C-IV (HAR-4525).

\section{HAR-4527. RR278}

$$
2110 \pm 80
$$

Cereals AML 811687 , from base of grain storage pit $\left(51^{\circ} 30^{\prime} 15^{\prime \prime} \mathrm{N}, 0^{\circ}\right.$ $22^{\prime} 24^{\prime \prime}$ E, Natl Grid Ref TQ 647811). Coll March 1980 and subm June 1981 by T J Wilkinson. Comment (TJW): ceramics from deposit agree better with replicate sample HAR-4635.

HAR-4635. RR278

$$
\begin{array}{r}
2350 \pm 70 \\
\delta^{\prime 3} C=-24.0 \%
\end{array}
$$

Cereals, AML 813338, from layer at base of Iron Age pit at Rectory Rd, Orsett (5 $1^{\circ} 30^{\prime} 15^{\prime \prime} \mathrm{N}, 0^{\circ} 22^{\prime} 24^{\prime \prime}$ E, Natl Grid Ref TQ 647811). Coll Spring 1980 by T J Wilkinson and subm Nov 1980 by P Murphy. Comment (PM): well sealed within pit containing assemblage of almost complete early Iron Age pottery. From same context as HAR-4527.

\section{HAR-4634. NS0600C}

$3280 \pm 70$

$\delta^{i 3} \mathrm{C}=-25.1 \%$

Charcoal, AML 813644, from prehistoric cremation pit, context $0600 \mathrm{C}$, site TQ $98 / 24$ at North Shoebury, Essex $\left(51^{\circ} 32^{\prime} 21^{\prime \prime} \mathrm{N}, 0^{\circ} 47^{\prime} 3^{\prime \prime} \mathrm{E}\right.$, Nati Grid Ref TQ 931860). Coll by J J Wymer and subm Aug 1981 by P Murphy. Comment (PM): result accords well with Middle Bronze Age enclosures and settlement features nearby.

\section{HAR-4843. 813339}

$$
\delta^{13} C=\begin{array}{r}
2100 \pm 80 \\
=-25.4 \%
\end{array}
$$

Charcoal, AML 813339 , from post hole, context 84 at Heybridge, Essex ( $51^{\circ} 44^{\prime} 30^{\prime \prime} \mathrm{N}, 0^{\circ} 40^{\prime} 49^{\prime \prime} \mathrm{E}$, Natl Grid Ref TL 85020823). Coll 1972 by P J Drury and subm by N P Wickenden. Comment (NPW): sample probably burned in silu in post hole of Iron Age four-post structure. See Wickenden and Drury (1986) for description of site. 


\section{Brixworth series}

Wood from All Saint's Church, Brixworth, Northamptonshire $\left(52^{\circ} 20^{\prime}\right.$ $0^{\prime \prime} \mathrm{N}, 0^{\circ} 54^{\prime} 13^{\prime \prime} \mathrm{W}$, Natl Grid Ref SP 747712). Coll Nov 1981 by N Wilson and subm April 1982 by M Audovy.

\section{HAR-4945. BX81705}

$1130 \pm 70$

AML 822326, from base courses of jamb of nave arcading.

\section{HAR-4946. BX81600}

$1270 \pm 80$

AML 822325, from just below a thin layer of mortar. Comment (MA): sample from probable construction layer and thus assoc with building of church.

\section{Raunds series}

Samples from Anglo-Saxon site at Raunds, Northamptonshire $\left(52^{\circ} 20^{\prime}\right.$ $54^{\prime \prime} \mathrm{N}, 0^{\circ} 32^{\prime} 0^{\prime \prime} \mathrm{W}$, Natl Grid Ref SP 999733). All samples except HAR4903 subm April 1982 by A Boddington, Northamptonshire Co Council. For description of site, see Boddington and Cadman (1981).

HAR-4903. 223

$$
\delta^{33} C=-25.4 \%
$$

Charcoal, AML 813690, from fill of possible pit oven, Site 77. Coll by S A Power and C Addison-Jones and subm Sept 1981 by G Cadman. Comment (GC): sample from 1st phase of Saxon occupation. Forms part of earliest period, represented by scatter of isolated features (Cadman, 1983).

\section{HAR-5010. 5222-111}

$$
970 \pm 70
$$

Human bone, from Angio-Saxon cemetery. Coll July 1979 and subm April 1982 by A Boddington. Comment (AB): sample contemporary with period IIC church.

HAR-5011. 5299-136

$$
\delta^{\prime 3} C=\begin{array}{r}
960 \pm 60 \\
-22.1 \%
\end{array}
$$

Human bone, from late Anglo/Saxon Christian cemetery. Coll July 1980 and subm April 1982 by A Boddington. Comment (AB): sample is contemporary with period II A/B church.

HAR-5012. 5286-135

$$
1000 \pm 70
$$

Human bone, from late Anglo/Saxon Christian cemetery. Coll July 1980 and subm April 1982 by A Boddington. Comment (AB): sample is contemporary with period II $\mathrm{A} / \mathrm{B}$ church. 
HAR-5013. 5266-122

Human bone, from late Anglo-Saxon Christian cemetery. Coll Oct 1979 and subm April 1982 by A Boddington. Comment (AB): sample is contemporary with period II A/B church.

HAR-5014. 5223-112

$1110 \pm 90$

$\delta^{13} C=-22.4 \%$ o

Human bone, from late Anglo-Saxon Christian cemetery. Coll July 1980 and subm April 1982 by A Boddington. Comment (AB): sample is contemporary with period II A/B church.

HAR-5015. 5015-71

$1040 \pm 70$

$\delta^{13} \mathrm{C}=-26.0 \%$

Charcoal from chancel of late Anglo/Saxon church. Coll July 1980 and subm April 1982 by A Boddington. Comment (AB): sample antedates period II C rebuilding.

HAR-5016. 5254-119

$$
\begin{array}{r}
1080 \pm 70 \\
\delta^{13} C=-22.6 \%
\end{array}
$$

Human bone, from late Anglo-Saxon cemetery. Coll Aug 1979 and subm April 1982 by A Boddington. Comment (AB): sample contemporary with period IIC church.

HAR-5019. 5178-113

$$
\begin{array}{r}
\mathbf{9 3 0} \pm \mathbf{7 0} \\
\delta^{13} C=-22.0 \% 0
\end{array}
$$

Human bone, from late Anglo/Saxon Christian cemetery. Coll May 1979 and subm April 1982 by A Boddington. Comment (AB): sample is contemporary with period IIC church.

HAR-5020. 5298-134

$$
\begin{array}{r}
\mathbf{1 1 0 0} \pm \mathbf{8 0} \\
\delta^{13} C=-22.8 \%
\end{array}
$$

Human bone, from late Anglo-Saxon cemetery. Coll July 1979 and subm April 1982 by A Boddington. Comment (AB): sample contemporary with period IIA/B church.

General Comment (GC \& AB): excavations at Raunds site "Furnells" include Saxon settlement, late Saxon church and cemetery and medieval manor house. Work on this began in 1977 and was completed in 1982 although excavation of surrounding sites continues to investigate nature and evolution of Saxon and Medieval landscape complex.

\section{Hullbridge series}

Samples from survey at Hullbridge basin, Essex. Coll June 1982 and subm Aug 1982 by P Murphy, except where indicated, on behalf of Essex Co Council. 
HAR-5221. H11ROOT

Wood, AML 823047, from tree roots in exposed estuarine clay surface at Site $11\left(51^{\circ} 38^{\prime} 11^{\prime \prime} \mathrm{N}, 0^{\circ} 36^{\prime} 26^{\prime \prime} \mathrm{E}\right.$, Natl Grid Ref TQ 80419634). Comment (PM): sample within area of prehistoric occupation exposed by machining (Murphy \& Wilkinson, 1982).

\section{HAR-5222. H1WOOD14}

$$
\begin{array}{r}
\mathbf{2 7 3 0} \pm \mathbf{6 0} \\
\delta^{13} C=-28.3 \% 0
\end{array}
$$

Wood, AML 823048 , from layer sealed by ca $2.0 \mathrm{~m}$ of estuarine clay at site 1 (51 $38^{\circ} 15^{\prime \prime} \mathrm{N}, 0^{\circ} 35^{\prime} 47^{\prime \prime} \mathrm{E}$, Natl Grid Ref TQ 79649645). Comment (PM): sampled brushwood, exposed by erosion, assoc with probable prehistoric platform structure (Murphy \& Wilkinson, 1982).

\section{HAR-5223. H4LPEATW}

$$
\begin{array}{r}
\mathbf{3 6 6 0} \pm \mathbf{7 0} \\
\delta^{13} C=-28.0 \% \text { o }
\end{array}
$$

Wood, AML 823049, from top of Flandrian lower peat at site $4\left(51^{\circ}\right.$ $37^{\prime} 48^{\prime \prime} \mathrm{N}, 0^{\circ} 36^{\prime} 17^{\prime \prime} \mathrm{E}$, Natl Grid Ref TQ 80269561). Comment (PM): agrees well with ${ }^{14} \mathrm{C}$ determination (HAR-5226) from base of lower peat at same loc (Murphy \& Wilkinson, 1982).

HAR-5224. H9UPEAT

$1500 \pm 70$

$\delta^{13} C=-29.1 \%$

Soil, AML 823050, from Flandrian upper peat at site $9\left(51^{\circ} 38^{\prime} 14^{\prime \prime} \mathrm{N}\right.$, $0^{\circ} 39^{\prime} 34^{\prime \prime} \mathrm{E}$, Natl Grid Ref TQ 84019657) described in Murphy and Wilkinson (1982).

HAR-5225. H4UPEATB

$$
\begin{array}{r}
1610 \pm 70 \\
\delta^{13} C=-30.3 \% 0
\end{array}
$$

Soil, AML 823051, from base of Flandrian upper peat at site $4\left(51^{\circ} 37^{\prime}\right.$ $48^{\prime \prime} \mathrm{N}, 0^{\circ} 36^{\prime} 17^{\prime \prime} \mathrm{E}$, Natl Grid Ref TQ 80269561) described in Murphy and Wilkinson (1982).

HAR-5226. H4LPEATB

$$
\begin{array}{r}
\mathbf{3 7 6 0} \pm \mathbf{7 0} \\
\delta^{13} \mathrm{C}=-30.0^{0} \%
\end{array}
$$

Soil, AML 823052, from base of Flandrian lower peat at site $4\left(51^{\circ} 37^{\prime}\right.$ $48^{\prime \prime} \mathrm{N}, 0^{\circ} 36^{\prime} 17^{\prime \prime} \mathrm{E}$, Natl Grid Ref TQ 80269561) described in Murphy and Wilkinson (1982).

\section{HAR-5227. H8ROOTS}

$$
\begin{array}{r}
4100 \pm 70 \\
\delta^{13} C=-30.6 \% 0
\end{array}
$$

Wood, AML 823053 , id. as tree roots, from site $8,40 \mathrm{~m} \mathrm{~S}$ of shore of Crouch Estuary $\left(51^{\circ} 38^{\prime} 14^{\prime \prime} \mathrm{N}, 0^{\circ} 39^{\prime} 2^{\prime \prime}\right.$ E, Natl Grid Ref TQ 83409653). Coll June 1978. Comment (PM): roots comprise part of currently exposed Flandrian submerged forest once sealed by estuarine clays, but sample measured was sub-aerially exposed (Murphy \& Wilkinson, 1982). 
HAR-5549. H44TWIGS

$$
\delta^{13} C=\begin{array}{r}
\mathbf{2 7 0} \pm \mathbf{8 0} \\
-28.5 \% o
\end{array}
$$

Wood, AML 831123, from context 44 of structure thought originally to be prehistoric $\left(51^{\circ} 37^{\prime} 54^{\prime \prime} \mathrm{N}, 0^{\circ} 38^{\prime} 3^{\prime \prime} \mathrm{E}\right.$, Natl Grid Ref TQ 82299589). Coll Dec 1982 by T J Wilkinson and subm April 1983. Comment (PM): 2nd sample from same structure (post H45, HAR-5550, below) yielded similar post-Medieval date; it is evident structure was part of old sea wall or related to it (Wilkinson \& Murphy, 1983a).

\section{HAR-5550. H45POST}

$$
\delta^{13} C=\begin{array}{r}
\mathbf{3 0 0} \pm \mathbf{9 0} \\
-25.2 \%
\end{array}
$$

Wood, AML 831124, from context 44 of structure thought originally to be prehistoric (51 $37^{\prime} 54^{\prime \prime} \mathrm{N}, 0^{\circ} 38^{\prime} 3^{\prime \prime} \mathrm{E}$, Natl Grid Ref TQ 82299589). Coll Dec 1982 by T J Wilkinson and subm April 1983.

\section{HAR-5732. H56/97}

$$
\delta^{13} C=\begin{array}{r}
180 \pm \mathbf{8 0} \\
-29.4 \% o
\end{array}
$$

Peat, AML 832738, from river bank at site 56, Canewdon $\left(51^{\circ} 37^{\prime} 43^{\prime \prime}\right.$ N, $0^{\circ} 46^{\prime} 51^{\prime \prime} \mathrm{E}$, Natl Grid Ref TQ 92449591). Coll June 1983 by T J Wilkinson. Comment (PM): upper peat layer result of inning during last few hundred years (Wilkinson \& Murphy, 1983b).

\section{HAR-5733. H2/91}

$$
\begin{array}{r}
\mathbf{3 0 2 0} \pm \mathbf{9 0} \\
\delta^{13} C=-25.3 \%
\end{array}
$$

Charcoal, AML 832739, from prehistoric salt working hearth at site 2, South Woodham Ferrers (51 $38^{\prime} 15^{\prime \prime} \mathrm{N}, 0^{\circ} 36^{\prime} 16^{\prime \prime}$ E, Natl Grid Ref TQ 80209646). Coll June 1983 by T J Wilkinson. Comment (PM): conforms well with late Bronze Age to early Iron Age pottery (Wilkinson \& Murphy, 1983b).

\section{HAR-5734. H29/67}

$$
\begin{array}{r}
\mathbf{2 9 5 0} \pm \mathbf{7 0} \\
\delta^{13} C=-25.9 \%
\end{array}
$$

Wood, AML 832740, id. as Quercus sp and Prunus sp, brushwood platform in base of clays of Holocene marine transgression from site $29\left(51^{\circ} 38^{\prime}\right.$ 27" N, $0^{\circ} 44^{\prime} 25^{\prime \prime}$ E, Natl Grid Ref TQ 89599717). Coll April 1983 by T J Wilkinson. Comment (PM): from platform on edge of estuary after Neolithic occupation ceased (Wilkinson \& Murphy, 1983b).

\section{HAR-5735. H29/68}

$$
\begin{array}{r}
\mathbf{3 2 5 0} \pm \mathbf{9 0} \\
\delta^{13} C=-25.0 \% 0
\end{array}
$$

Wood, AML 832741, id. as Fraxinus sp, from part of wooden trackway from site 29 (51 $38^{\prime} 27^{\prime \prime}$ N, $0^{\circ} 44^{\prime} 25^{\prime \prime}$ E, Natl Grid Ref TQ 89599717). Coll April 1983 by T J Wilkinson. Comment (PM): from platform on edge of estuary after Neolithic occupation ceased (Wilkinson \& Murphy, 1983a,b). 
HAR-5736. H22WOOD

Wood, AML 832742, from group of timbers assoc with hurdles at site $22\left(51^{\circ} 38^{\prime} 21^{\prime \prime} \mathrm{N}, 0^{\circ} 46^{\prime} 8^{\prime \prime} \mathrm{E}\right.$, Natl Grid Ref TQ 91579707). Coll April 1983 by $\mathrm{T} \mathrm{J}$ Wilkinson. Comment (PM): from hurdle structure contained within marine transgression clays stratigraphically later than lower peats which have ${ }^{14} \mathrm{C}$ age $2000-1700$ BC (Wilkinson \& Murphy, 1983b).

\section{HAR-5737. H23WOOD}

$$
3680 \pm 70
$$
at at site $23\left(51^{\circ} 38^{\prime} 5^{\prime \prime} \mathrm{N}, 0^{\circ} 46^{\prime} 42^{\prime \prime} \mathrm{E}\right.$, Natl Grid Ref TQ 92249660). Coll April 1983 by T J Wilkinson. Comment (PM): stratigraphically contemporary with lower peat, conforming with ${ }^{14} \mathrm{C}$ determinations on lower peat from elsewhere in basin (Wilkinson \& Murphy, 1983a).

General Comment (PM): aim of survey is to locate and describe archaeol sites currently being eroded in valleys of $\mathrm{R}$ Crouch and $\mathrm{R}$ Blackwater and to relate these to sediment sequences. ${ }^{14} \mathrm{C}$ is used for main chronological framework. Results from Crouch estuary fall into 4 groups: 1) wood and other organic material in Lower Peat, ca 3660 to $4100 \mathrm{BP}, 2)$ landing stage structures and salt-working sites assoc with Middle Clay, ca 2620 to 3250 BP, 3) Upper (Phragmites) Peat ca, 1500 to 1610 BP, and 4) deposits and structures assoc with reclamation following renewed sedimentation (Upper Clay), ca 270 to $180 \mathrm{BP}$.

\section{Canterbury series}

Two charcoal samples, from floor of Late Saxon cellared building cut into natural at 69 a Stour St, Canterbury, Kent $\left(51^{\circ} 16^{\prime} 38^{\prime \prime} \mathrm{N}, 1^{\circ} 4^{\prime} 49^{\prime \prime} \mathrm{E}\right.$, Natl Grid Ref TR 148577). Coll 1981 by T Tatton-Brown and subm by D Haddon-Reece.

HAR-5229. STOUR02

$$
\begin{array}{r}
1060 \pm 70 \\
\delta^{13} C=-26.5 \% \text { o }
\end{array}
$$

AML 822359. Comment (TTB): excavators ref CBR V 246A.

HAR-5230. STOUR03

$$
1170 \pm 70
$$

AML 822360. Comment (TTB): excavators ref CBR V 246B.

General Comment (TTB): samples overlay deposit of occupation loam, may be charred timber from destruction of building. Sample was sealed by dark loam infill, containing carbon, clay, and daub. One other sample, fragment of preserved and waterlogged timber (AML 822361), part of vertical post, produced date $1270 \pm 70$ (Bennett, Tatton-Brown \& Blockley, 1980). 


\section{Rowden series}

Samples from Neolithic and Bronze Age contexts at Rowden, Dorset. Coll Sept 1981 by P J Woodward and subm by D Haddon-Reece. See Woodward (1981) for description of site.

HAR-5245. RD82287 $4690 \pm 70$

Charcoal, AML 822635, from layer 287.

$$
\delta^{13} C=-26.5 \%
$$

HAR-5247. RD82571

$4940 \pm 70$

$\delta^{13} C=-26.7 \% 0$

Charcoal, AML 822637, from layer 571. Subm Oct 1982.

HAR-5248. RD82634

$4860 \pm 70$

$\delta^{13} \mathrm{C}=-26.5 \%$

Charcoal, AML 822638, from layer 634. Subm Oct 1982.

HAR-5548. RD815773

$$
\begin{array}{r}
2940 \pm 70 \\
\delta^{13} C=-23.6 \% 0
\end{array}
$$

Carbonized grain, AML 831125, from storage pit in Middle Bronze Age hut. Subm April 1983. Comment (PJW): result compares well with HAR-5698, from same pit.

HAR-5698. RD815773

$$
\begin{array}{r}
\mathbf{2 9 2 0} \pm \mathbf{8 0} \\
\delta^{13} C=-23.2 \% 0
\end{array}
$$

Charcoal, AML 831125, from storage pit in Middle Bronze Age hut. Subm April 1983. Comment (PJW): result as expected on archaeol evidence; site compares with Black Patch excavation, Sussex (Drewett, 1982).

General Comment (PJW): HAR-5245, -5247, -5248, assoc with large post pit, confirm early Neolithic date antedating Late Bronze Age hut and settlement complex.

\section{Winwick series}

Charcoal from Southworth Hall Barrow, Winwick, Cheshire $\left(53^{\circ} 26^{\prime}\right.$ $15^{\prime \prime} \mathrm{N}, 2^{\circ} 34^{\prime} 25^{\prime \prime}$ W, Natl Grid Ref SJ 619936). Coll Sept/Oct 1980 and subm Oct 1981 by D Freke.

HAR-5258. 14980112

$$
\begin{array}{r}
3390 \pm \mathbf{7 0} \\
\delta^{13} \mathrm{C}=-26.7 \% 0
\end{array}
$$

AML 824012, from context 84, fill of cremation pit in fill of 2nd phase ditch of 2nd phase Barrow. Coll Oct 1980. Comment (DF): sample assoc with collared urn and accessory vessel.

\section{HAR-5259. 14980130}

$$
\begin{array}{r}
\mathbf{4 0 9 0} \pm \mathbf{8 0} \\
\delta^{I 3} C=-27.2 \% 0
\end{array}
$$

AML 824014, from context 327, large pit filled with burned stones, charcoal, and Bronze Age pottery fragments. Comment (DF): pit was $10 \mathrm{~m}$ 
outside outer ditch of 2nd phase Barrow. Earliest result from 1st phase of Barrow was HAR-5262, below.

\section{HAR-5260. 14980141}

$3630 \pm 70$ dith of inner ditch of phase 2 Barrow. Comment (DF): sample not burned in situ but dumped, one of several patches.

\section{HAR-5261. 14980145}

$3470 \pm 70$

AML 824016, from thick charcoal layer under 2nd phase mound. Comment (DF): interpreted as burned mortuary structure.

HAR-5262. 14980159

$$
\begin{array}{r}
\mathbf{3 6 9 0} \pm \mathbf{8 0} \\
\delta^{13} C=-27.6 \% 0
\end{array}
$$

AML 824017, from context 921, small pit under 1st phase mound of 2nd phase Barrow. Comment (DF): earliest date for Barrow.

\section{Somerset Levels series}

Following 34 dates were coll during excavations between 1974 and 1984 from Somerset Levels sites, Somerset. Following general dates, main series was divided into sub-series (Meare Lake Village, East Moors, Eclipse Site, Sedgemoor, and Sweet Track). For introduction to project and other results, see Harwell II (R, 1977, v 19, no. 3, p 415-416); III (R, 1979, v 21 , no. 3, p 360-364); IV (R, 1985, v 27, no. 1, p 77-80); V (R, 1987, v 29, no. 1, p 87-92). Except where noted, all samples coll and subm by J M Coles, Dept Archaeol, Cambridge.

\section{HAR-1490. SLP76-2}

$$
\begin{array}{r}
3200 \pm \mathbf{1 3 0} \\
\delta^{13} C=-26.8 \%
\end{array}
$$

Wood, AML 760302, from rings 68 to 80 of 118 -year floating treering sequence at Meare Lake track, Somerset. Coll 1974-5 and subm Jan 1976 by R Morgan. Comments: small sample due to experimental difficulties accounts for larger than normal error term. (JMC): 2nd sample in dendrochronologic series of 3 . Includes sections of 4 timbers. Wood was distorted due to peat drying out, some root intrusion. Also see HAR-943, Harwell III (R, 1979, v 21, no. 3, p 360).

\section{HAR-2772. SLP7809}

$$
3270 \pm 70
$$

Wood, from terminal of Bronze Age trackway at terminal site 1, Shapwick, Somerset. Coll and subm July 1978. Comment (JMC): complex of wood.

\section{HAR-3446. SLP7918}

$$
\begin{array}{r}
\mathbf{2 6 3 0} \pm \mathbf{8 0} \\
\delta^{13} C=-27.7 \% 0
\end{array}
$$

Charcoal, AML 79476 from Withy Bed Copse, Shapwick, Somerset $\left(51^{\circ} 8^{\prime} 50^{\prime \prime} \mathrm{N}, 2^{\circ} 48^{\prime} 20^{\prime \prime} \mathrm{W}\right.$, Natl Grid Ref ST 435394). Coll by C R Sturdy 
and subm Aug 1979. Also see HAR-994, Harwell III (R, 1979, v 21, no. 3, p 361).

HAR-3838. SLP8009

$3600 \pm 70$

Wood from early Bronze Age hurdle structure at Eclipse track, Somerset. Coll by B J Orme and subm April 1980.

HAR-4078. SLP8010

$$
\begin{array}{r}
\mathbf{4 8 8 0} \pm \mathbf{1 0 0} \\
\delta^{13} C=-27.4 \%
\end{array}
$$

Wood, from newly discovered track structure in peat at Bisgrove, Somerset. Coll Sept 1980 and subm Oct 1980.

\section{HAR-4130. SLP8011}

$$
\begin{array}{r}
\mathbf{4 2 8 0} \pm \mathbf{7 0} \\
\delta^{13} \mathrm{C}=-28.0 \% 0
\end{array}
$$

Peat from SF 80.37 at Decoy Pool Wood, Shapwick, Somerset. Coll Oct 1980 and subm Nov 1980. Comment (JMC): peat in direct assoc with Neolithic stone axe.

\section{HAR-4999. SLP8205}

$$
\begin{array}{r}
\mathbf{5 4 2 0} \pm \mathbf{7 0} \\
\delta^{13} \mathrm{C}=-28.0^{0} \%
\end{array}
$$

Peat, from West Edington Valley, Somerset $\left(51^{\circ} 10^{\prime} 41^{\prime \prime} \mathrm{N}, 2^{\circ} 52^{\prime} 27^{\prime \prime}\right.$ W, Natl Grid Ref ST 389425). Coll and subm April 1982. Comment (JMC): sample used to date clay-peat interface in W Brue.

\section{Meare Lake Village series}

Samples from Meare Lake Village West, Somerset $\left(51^{\circ} 10^{\prime} 33^{\prime \prime} \mathrm{N}, 2^{\circ}\right.$ $47^{\prime} 38^{\prime \prime}$ W, Natl Grid Ref ST 445422). For description of site, see Orme et al (1981). For other dates in series, see Harwell IV (R, 1985, v 27, no. 1, p $79-80)$.

\section{HAR-3489. SLP7918}

$$
\begin{array}{r}
2200 \pm 70 \\
\delta^{13} C=-29.7 \% 0
\end{array}
$$

Wood, AML 794485, from planking in gully between mounds. Coll and subm Sept 1979. Comment (JMC): series of stratified horizons of peat, wood occupation debris marking several periods of activity assoc with bone, wood, bronze, iron, lead, pottery, glass, etc.

\section{HAR-3492. SLP7910}

$$
\begin{array}{r}
2130 \pm 60 \\
\delta^{13} C=-25.0 \% 0
\end{array}
$$

Wood, id. as plank of elm (decayed), AML 794477, assoc with Iron Age site. Coll and subm Sept 1979. Comment (JMC): series of stratified horizons of peat with occupation debris marking several phases of activity assoc with bone, wood, bronze, iron, lead, pottery, glass, etc.

\section{HAR-3535. SLP7915}

$$
\begin{array}{r}
2250 \pm 70 \\
\delta^{13} C=-26.8 \%
\end{array}
$$

Peat, AML 794482, from beneath central floor, assoc with large storage vessel. Coll and subm Sept 1979. 
HAR-3546. SLP7911

$\mathbf{2 4 1 0} \pm \mathbf{8 0}$ Sept 1979.

HAR-3633. SLP7913

$$
\begin{array}{r}
\mathbf{2 7 0 0} \pm \mathbf{7 0} \\
\delta^{13} C=-29.2 \%
\end{array}
$$

Charcoal, AML 794480, from top of underlying peat surface, beneath occupation deposit. Coll and subm Sept 1979.

\section{HAR-3634. SLP7912}

$2230 \pm 60$

Peat, AML 794479, from black earth occupation deposit. Coll and subm Sept 1979.

\section{HAR-3693. SLP8004}

$$
\begin{array}{r}
\mathbf{2 1 7 0} \pm \mathbf{7 0} \\
\delta^{13} C=-23.3 \% 0
\end{array}
$$

Wood, id. as elm coll Sept 1979 and subm Feb 1980. Comment (JMC): assoc with hearth to NW of occupation floor.

HAR-3719. SLP7918

$$
\begin{array}{r}
\mathbf{2 1 9 0} \pm \mathbf{7 0} \\
\delta^{13} C=-28.0 \%
\end{array}
$$

Wood, AML 794481, from remains of wood under central floor of occupation site. Coll and subm Sept 1979.

HAR-3740. SLP8002

$$
\begin{array}{r}
\mathbf{2 8 1 0} \pm \mathbf{7 0} \\
\delta^{13} C=-29.6 \% \text { o }
\end{array}
$$

Wood from peat surface, NW of main occupation floor. Coll Sept 1979 and subm Feb 1980

HAR-3744. SLP8003

$$
\begin{array}{r}
\mathbf{2 2 8 0} \pm \mathbf{8 0} \\
\delta^{13} C=-27.3 \%
\end{array}
$$

Wood and peat, from occupation surface. Coll Sept 1979 and subm Feb 1980 .

HAR-3745. SLP8001

$$
2080 \pm 60
$$$$
\delta^{13} C=-28.5 \%
$$
1980.

Soil, from central floor of occupation. Coll Sept 1979 and subm April

HAR-3864. SLP8005

$$
\begin{array}{r}
\mathbf{2 3 7 0} \pm \mathbf{7 0} \\
\delta^{13} C=-28.6 \% 0
\end{array}
$$

Peat, from monolith top 2.98 to $3.00 \mathrm{~m}$. Coll Sept 1979 by A E Caseldine and subm April 1980. Comment (JMC): a few very fine rootlets in samples. 
HAR-3891. SLP8006

Wood from monolith, 3.04 to $3.06 \mathrm{~m}$. Coll Sept 1979 by A E Caseldine and subm April 1980.

HAR-3892. SLP8007

$$
1870 \pm 80
$$

Charcoal, id. as peat/charcoal, from monolith 3.10 to $3.12 \mathrm{~m}$ occupation deposit. Coll Sept 1979 by A E Caseldine and subm April 1980.

HAR-3896. SLP8008

$2220 \pm 90$

$\delta^{13} \mathrm{C}=-27.8 \%$

Wood, from monolith, 3.14 to $3.16 \mathrm{~m}$, occupation deposit. Coll Sept 1979 by A E Caseldine and subm April 1980.

\section{East Moors series}

Wood samples from East Moors, Ashcott Heath, Somerset. Coll by C R Sturdy and subm Aug 1979.

HAR-3448. SLP7906

$$
\begin{array}{r}
\mathbf{3 7 7 0} \pm \mathbf{7 0} \\
\delta^{13} C=-30.8 \% 0
\end{array}
$$

From site 3.

\section{HAR-3449. SLP7908}

$$
\begin{array}{r}
\mathbf{3 7 5 0} \pm \mathbf{7 0} \\
\delta^{13} C=-29.3 \%
\end{array}
$$

From area 10.

HAR-3447. SLP7907

$$
\begin{array}{r}
\mathbf{3 8 7 0} \pm \mathbf{8 0} \\
\delta^{13} C=-29.0 \% 0
\end{array}
$$

From area 6.

\section{Eclipse site series}

Peat samples from Eclipse track site, Meare Heath, Somerset $\left(51^{\circ} 9^{\prime}\right.$ $41^{\prime \prime} \mathrm{N}, 2^{\circ} 47^{\prime} 17^{\prime \prime} \mathrm{W}$, Natl Grid Ref ST 449406). Coll Sept 1980 by A E Caseldine and subm Feb 1982. For description of zones, see Beckett and Hibbert (1979) and Orme (1982); for other dates in series, see Harwell V (R, 1987, v 29, no. 1, p 87-92).

\section{HAR-4866. SLP8110}

$$
\begin{array}{r}
\mathbf{4 7 8 0} \pm \mathbf{7 0} \\
\delta^{13} C=-28.9 \%
\end{array}
$$

From lower division of peat monolith, dating E1.E2 boundary and therefore, region A/B boundary.

HAR-4867. SLP8111

$3840 \pm 60$

$$
\delta^{13} C=-29.3 \%
$$

From within zone E4, of local sequence, probably near or at regional zone $\mathrm{D} / \mathrm{E}$ boundary (E80.54). 
HAR-4868. SLP8112

$3240 \pm 70$

From immediately below hurdle track, near top of monolith (E80.26). Comment (JMC): possible modern rootlet contamination.

\section{Sedgemoor series}

Wood, from prehistoric structure, Sedgemoor, Somerset. Coll by K Campbell and subm April 1981.

HAR-4374. SLP8101

From site $81.6 \mathrm{~A}$.

HAR-4375. SLP8102

From site $81.6 \mathrm{~B}$.

$$
\begin{array}{r}
\mathbf{4 5 1 0} \pm \mathbf{8 0} \\
\delta^{13} C=-28.6 \% 0
\end{array}
$$

$4690 \pm 90$

$\delta^{13} C=-28.3 \%$

\section{Sweet Track series}

Peat, from regional pollen assemblage zone boundaries from Sweet Track, Somerset. Coll Sept 1980 by A E Caseldine and subm Nov 1982. For other dates in series, see Harwell III (R, 1979, v 21, no. 3, p 363-364).

\section{HAR-5294. SLP8209}

From pollen boundary C/D.

\section{HAR-5295. SLP8208}

From pollen boundary B/C.

\section{HAR-5296. SLP8207}

From pollen boundary A/B.

\section{HAR-4540. 41-149}

Charcoal, AML 8110725, from Trelan 2, Cornwall. Coll by G H Smith and subm Aug 1981 by N D Balaam.

\section{Balksbury Camp series}

Samples from Iron Age hillfort at Balksbury Camp, Andover, Hampshire $\left(51^{\circ} 11^{\prime} 56^{\prime \prime} \mathrm{N}, 1^{\circ} 29^{\prime} 51^{\prime \prime} \mathrm{W}\right.$, Natl Grid Ref SU 351446). Coll 1981 by $\mathrm{K}$ Smith and subm Aug 1982 by N D Balaam. Comment: for other dates in series, see Harwell II (R, 1977, v 19, no. 3, p 408).

HAR-5124. 36-326

$$
\begin{array}{r}
\mathbf{3 5 3 0} \pm \mathbf{8 0} \\
\delta^{13} C=-23.5 \% 0
\end{array}
$$


HAR-5127. 36-1465

$$
\begin{array}{r}
\mathbf{2 8 0 0} \pm \mathbf{7 0} \\
\delta^{13} C=-25.9 \% \text { o }
\end{array}
$$

Charcoal, id. as mostly Fraxinus sp, with small quantities of Populus sp, Fagus sp, and Quercus sp, AML 8112890, from small pit in hillfort with early Iron Age pottery.

\section{Catterick series}

Human bone from Catterick (Natl Grid Ref SE 240972). Coll Oct 1981 by P Wilson and subm Oct 1982 by N D Balaam.

HAR-5272. 46-941

$1640 \pm 70$

AML 8111678 .

$\delta^{13} C=-22.5 \%$

HAR-5273. 46-1742

$1660 \pm 70$

AML 8111679 .

$\delta^{13} C=-23.5 \%$

HAR-5275. 46-1732

$\delta^{13} C=-22.0^{\%}$

AML 8111564 .

HAR-5276. 46-709

$1500 \pm 70$

AML 8111254.

$\delta^{13} C=-21.9 \%$

HAR-5277. 46-3520

$1560 \pm 70$

AML 8111950.

$\delta^{13} C=-22.5 \% 0$

HAR-5740. 46-1033

$1900 \pm 80$

$\delta^{13} C=-23.6 \%$

AML 8111316, from crouched burial pit with no artifacts. Comment (NDB): burial sealed by early Roman features.

\section{Ardleigh series}

Charcoal, from possibly Bronze Age site at Ardleigh, Essex. Coll by J Hinchliffe and subm by N D Balaam.

HAR-3908. 29-750

$3600 \pm 80$

AML 7911015, id. as Quercus sp, from mature timber and Corylus avellana nut shell, from Bronze Age cremation. Subm June 1980.

HAR-5126. 29-1939

$$
\begin{array}{r}
\mathbf{2 8 7 0} \pm \mathbf{8 0} \\
\delta^{13} C=-25.8 \% 0
\end{array}
$$

AML 7910872, id. as Quercus sp, from lower fill of ditch enclosure. Coll 1976 and subm Aug 1982. Comment (NDB): possibly late Bronze Age. 
HAR-5128. 29-1937

$2940 \pm 70$

$\delta^{13} C=-26.0^{\circ} \%$

AML 7910865, id. as Quercus sp, from lower fill of ditch. Coll 1979 and subm Aug 1982. Comment (NDB): possibly late Bronze Age.

HAR-5129. 29-7542

$$
\begin{array}{r}
\mathbf{3 0 5 0} \pm \mathbf{7 0} \\
\delta^{13} C=-27.1 \% 0
\end{array}
$$

AML 7911015, id. as Crataegus sp, from ditch of possible cursus. Coll 1979 and subm Aug 1982.

\section{Queensford Mill Cemetery series}

Human bone, from graves of late and sub-Roman cemetery at site I along rte of Dorchester By-Pass, Dorchester-on-Thames, Oxfordshire $\left(51^{\circ}\right.$ $38^{\prime} 59^{\prime \prime} \mathrm{N}, 1^{\circ} 9^{\prime} 32^{\prime \prime} \mathrm{W}$, Natl Grid Ref SU 58189493). Coll Spring 1981 and subm Nov 1982 by R A Chambers. Comment (RAC): there was no stratigraphic relationship between graves of extra-mural cemetery to $\mathrm{NE}$ of

\begin{tabular}{|c|c|c|}
\hline HAR-5323. & DBPIF34 & $\begin{array}{r}1480 \pm 70 \\
\delta^{13} C=-22.3 \% 0\end{array}$ \\
\hline HAR-5324. & DBPIF64 & $\begin{array}{r}1430 \pm 70 \\
\delta^{13} C=-24.4 \%\end{array}$ \\
\hline HAR-5350. & DBPIF48 & $\begin{array}{r}\mathbf{1 5 5 0} \pm \mathbf{7 0} \\
\delta^{13} C=-21.8 \%\end{array}$ \\
\hline HAR-5351. & DBPIF75 & $\begin{array}{r}1550 \pm 80 \\
\delta^{13} C=-23.1 \% 0\end{array}$ \\
\hline
\end{tabular}
Roman town.

\section{Church Lawton series}

Charcoal from Church Lawton, Alsager, Cheshire. Coll by R McNeil and subm May 1983 by R McNeil, Rescue Archaeol Comm, Univ Liverpool.

HAR-5533. 3551F12

$$
\begin{array}{r}
3600 \pm 100 \\
\delta^{13} C=-24.9^{0} \% 0
\end{array}
$$

AML 830768, from Bronze Age burial mound ( $53^{\circ} 5^{\prime} 52^{\prime \prime} \mathrm{N}, 2^{\circ} 17^{\prime} 12^{\prime \prime}$ W, Natl Grid Ref SJ 808557). Coll Jan 1983. Comment (RMcN): sample from possible mortuary house, representing only evidence that mound was ever a burial ground.

\section{HAR-5534. 3551F14}

$$
\begin{array}{r}
4100 \pm 160 \\
\delta^{13} C=-26.3 \% o
\end{array}
$$

AML 830769, from old ground surface sealed under barrow, $\left(53^{\circ} 5^{\prime}\right.$ $52^{\prime \prime}$ N, $2^{\circ} 17^{\prime} 12^{\prime \prime} \mathrm{W}$, Natl Grid Ref SJ 808557). Coll Jan 1983. Comments: small sample accounts for larger than normal error term. (RMcN): deposit assoc with previous and early phase of Barrow. 
HAR-5535. 3547F5

AML 830772, from fire pit used for cremating bones $\left(53^{\circ} 5^{\prime} 56^{\prime \prime} \mathrm{N}, 2^{\circ}\right.$ $17^{\prime} 12^{\prime \prime}$ W, Natl Grid Ref SJ 808558). Coll Aug 1982. Comment (RMcN): sample is sealed by turf and belongs to Phase 2 Barrow.

HAR-5536. 3547F11

$$
\begin{array}{r}
3300 \pm \mathbf{8 0} \\
\delta^{13} C=-26.9 \%
\end{array}
$$

AML 830773, from fire pit $\left(53^{\circ} 5^{\prime} 56^{\prime \prime} \mathrm{N}, 2^{\circ} 17^{\prime} 12^{\prime \prime} \mathrm{W}\right.$, Natl Grid Ref SJ 808558). Coll Aug 1982. Comment (RMcN): sample assoc with Phase 2 Barrow.

HAR-5537. 3547F12

$$
\begin{array}{r}
3490 \pm \mathbf{8 0} \\
\delta^{13} C=-26.3 \% 0
\end{array}
$$

AML 830774, from burial lid of primary insertion $\left(53^{\circ} 5^{\prime} 56^{\prime \prime} \mathrm{N}, 2^{\circ} 17^{\prime}\right.$ 12" W, Natl Grid Ref SJ 808558). Coll Aug 1982. Comment (RMcN): no skeleton present because soil was very acid; pit cuts old ground surface and is sealed by sand of Phase 1 Barrow.

HAR-5538. 3547F13

$$
\begin{array}{r}
\mathbf{3 5 6 0} \pm \mathbf{8 0} \\
\delta^{13} C=-24.5 \% 0
\end{array}
$$

AML 830775, from ditch surrounding barrow $\left(53^{\circ} 5^{\prime} 56^{\prime \prime} \mathrm{N}, 2^{\circ} 17^{\prime} 12^{\prime \prime}\right.$ W, Natl Grid Ref SJ 808558). Coll Aug 1982. Comment (RMcN): ditch antedates construction of phase I Barrow and is sealed by slumping from Barrow.

HAR-5539. 3547F16

$$
\begin{array}{r}
3500 \pm 100 \\
\delta^{13} C=-26.6 \% 0
\end{array}
$$

AML 830776, from inside accessory urn $\left(53^{\circ} 5^{\prime} 56^{\prime \prime} \mathrm{N}, 2^{\circ} 17^{\prime} 12^{\prime \prime} \mathrm{W}\right.$, Natl Grid Ref SJ 808558). Coll Sept 1982. Comment (RMcN): satellite burial, phase 1, feature sealed by sand of mound, no cremation present.

HAR-5541. 3547F26

$$
\begin{array}{r}
\mathbf{3 5 0 0} \pm \mathbf{8 0} \\
\delta^{13} C=-25.6 \% 0
\end{array}
$$

AML 830778, from pit containing accessory urn $\left(53^{\circ} 5^{\prime} 56^{\prime \prime} \mathrm{N}, 2^{\circ} 17^{\prime}\right.$ 12" W, Natl Grid Ref SJ 808558). Coll Sept 1982. Comment (RMcN): satellite burial, phase 1, no cremation present.

\section{HAR-5614. MK117040}

$1940 \pm 110$

$\delta^{13} C=-27.7 \%$

Wood, AML 823999, from four stakes at site MK117, context 95 at Mill Field site, Bow Brickhill Parish, Buckinghamshire $\left(52^{\circ} 0^{\prime} 25^{\prime \prime} \mathrm{N}, 0^{\circ} 42^{\prime}\right.$ 1" W, Natl Grid Ref SP 89213511). Coll by M Petchey and subm June 1983 by D Haddon-Reece. Comment (MP): context is 2nd lowest fill of ditch 4 in sec F of blue-gray sticky clay. Each stake at least $1.5 \mathrm{~m}$ long lying horizontally in base of ditch 4 (Loveday \& Petchey, 1982). 


\section{Coppergate Helmet series}

Wood from Coppergate Development, York, North Yorkshire $\left(53^{\circ} 57^{\prime}\right.$ $28^{\prime \prime} \mathrm{N}, 1^{\circ} 4^{\prime} 46^{\prime \prime} \mathrm{W}$, Natl Grid Ref SE 604517). Coll May 1982 by N F Pearson and subm Sept 1983 by A K G Jones, Environmental Archaeol Unit, Univ York.

HAR-5974. 82/22/68

$1160 \pm 70$

AML 834741, id. as ash (Fraxinus sp), from stake fragments of pit lining. Comment (AKGJ): pit was overlain by modern building rubble and some Medieval layers and was a few meters away from pit containing helmet.

HAR-5975. 82/22/72

$1250 \pm 70$

AML 834742, id. as oak (Quercus sp), from same fills as helmet.

General Comment (AKGJ): samples came from area in which Coppergate helmet was found. Results suggest helmet was buried during Anglian period (Addyman, Pearson \& Tweddle, 1982).

\section{REFERENCES}

Addyman, P V, Pearson, N F and Tweddle, D, 1982, The Coppergate helmet: Antiquity, v 56. p 189-194.

Allen D, 1981, The excavation of a Beaker burial monument at Ravenstone, Buckinghamshire, in 1978: Archaeol Jour, v 138, p 72-117.

Beckett, S C and Hibbert, F A, 1979, Vegetational change and the influence of prehistoric man in the Somerset Levels: New Phytol, v 83, p 577-600.

Bennett, P, Tatton-Brown, T and Blockley, K, 1980, Interim report on excavations in 1980 by the Canterbury Archaeological Trust: Archaeol Cantiana, v XCVI, p 396-410.

Boddington, A and Cadman, G, 1981, Raunds: an interim rept on excavations 1977-80: Anglo-Saxon studies in Archaeol and Hist 2: Brit Archaeol Rept, Br ser, v 92.

Cadman, G E, 1983, Raunds Furnells 1977-83, an excavation summary: Medieval Archacology, v 27, p 107-122.

Cadman, G E and Foard, G, 1984, Raunds-manorial and village origins, in Faul, M I., ed, Studies in Late Anglo-Saxon settlement: Oxford Univ Dept External Studies, p 81-100.

Dent, J S, 1978, Wetwang Slack: Current Archaeol, v 61, p 47-49. p 23-39. 1979, Bronze Age burials from Wetwang Slack: Yorkshire Archaeol Jour, v 51 , Wolds: Prehist Soc Proc, v 48, p 437-457.

1983, A summary of the excavations carried out in Garton Slack and Wetwang Slack 1964-1980: East Riding Archaeologist, v 7, p 1-4.

Drewett, P, 1982, Later Bronze Age downland economy and excavations at Black Patch, Fast Sussex: Prehist Soc Proc, v 48, p 321-400.

Durham, B G, 1977, Archaeol investigations in St Aldates, Oxford: Oxoniensia, v 42, p $174-$ 175 . v 13, p 138-140.

1984, The Thames crossing at Oxford: Oxoniensia, v 49, p 81-82.

Loveday, R and Petchey, M, 1982, Oblong ditches: a discussion and some new evidence: Acrial Archaeology, v 8, p 17-24

Martin, E, 1981, Archaeological excavations in Suffolk, 1980: Suffolk Inst Archacol Proc v 35 , pt 1 , p 79

Murphy, P and Wilkinson, T J, 1982, The Hullbridge Basin survey, interim rept: Fssex (i) Council, no 1.

Orme, B J, 1982, The use of radiocarbon dates from the Somerset Levels: Somerset I cevels Papers, no. 8, p 9-25. 
Orme, B J, Coles, J M, Caseldine, A E and Bailey, G N, 1981, Meare Lake Village West: Somerset Levels Papers, no. 7, p 12-69.

Otlet, R L, 1979, An assessment of errors in liquid scintillation counting, in Berger, R and Suess, $\mathrm{H}$ E, eds, Radiocarbon dating, Internatl ${ }^{14} \mathrm{C}$ conf, 9th, Proc: Berkeley, Univ California Press, p 256-267.

Otlet, R L and Walker, A J, 1983, The computer writing of radiocarbon reports and further developments in the storage and retrieval of archaeologic data, in Mook, W G and Waterbolk, $\mathrm{H} \mathrm{T}$, eds, ${ }^{14} \mathrm{C}$ and archaeology, Internatl symposium, 1st, Proc: PACT, v 8, p $91-$ 106.

Otlet, R L and Warchal, R M, 1978, Liquid scintillation counting of low-level ${ }^{14} \mathrm{C}$, in Crook, M A and Johnson, P, eds, Liquid scintillation counting, vol 5: London, Heyden, p 210-218.

Shoesmith, R, 1980, Rept of sectional recorders: Archaeol Trans Woolhope Nature \& Field Club, v 43, p 225-226.

1982, Excavations on and close to the defences, Hereford City Excavations, vol 2: Council Br Archaeol Research Rept no, 46, p 68-69.

Wickenden, N P and Drury, P J, 1986, Prehistoric settlement and the Romano-British small town at Heybridge, Essex: Essex Archaeol \& Hist, v 16, no. 2

Wilkinson, T J, 1980, Rescue archaeology along the A13 in S Essex: a preliminary report: Essex Co Council Rept, p 15-16.

Wilkinson, T J and Murphy, P, 1983a, The Hullbridge Basin survey, interim rept: Essex Co Council, no. 3 (3.2), p 10-12. p $1-5$.

1983b, The Hullbridge Basin survey, interim rept: Essex Co Council, no. 4,

oodward, P J, 1981, Excavations at Rowden-an interim note: Dorset Nat Hist Archaeol Soc Proc, v 103, p 119-121. 